\title{
MassARRAY-based simultaneous detection of hotspot somatic mutations and recurrent fusion genes in papillary thyroid carcinoma: the PTC-MA assay
}

\author{
Chiara Pesenti ${ }^{1,2}$ - Marina Muzza $^{2}$ - Carla Colombo ${ }^{2}$ - Maria Carla Proverbio ${ }^{2}$. \\ Claudia Farè $^{1} \cdot$ Stefano Ferrero ${ }^{1,3} \cdot$ Monica Miozzo ${ }^{1,2} \cdot$ Laura Fugazzola $\mathbb{D}^{2,4}$. \\ Silvia Tabano ${ }^{1,2}$
}

Received: 1 September 2017 / Accepted: 22 November 2017 / Published online: 6 December 2017

(C) The Author(s) 2017. This article is an open access publication

\begin{abstract}
Purpose We exploited the MassARRAY (MA) genotyping platform to develop the "PTC-MA assay", which allows the simultaneous detection of 13 hotspot mutations, in the BRAF, KRAS, NRAS, HRAS, TERT, AKT1, PIK3CA, and EIFlAX genes, and six recurrent genetic rearrangements, involving the RET and TRK genes in papillary thyroid cancer (PTC).

Methods The assay was developed using DNA and cDNA from 12 frozen and 11 formalin-fixed paraffin embedded samples from 23 PTC cases, together with positive and negative controls.

Results The PTC-MA assay displays high sensitivity towards point mutations and gene rearrangements, detecting their presence at frequencies as low as 5\%. Moreover, this technique allows quantification of the mutated alleles identified at each tested locus.
\end{abstract}

Electronic supplementary material The online version of this article (https://doi.org/10.1007/s12020-017-1483-2) contains supplementary material, which is available to authorized users.

Laura Fugazzola and Silvia Tabano contributed equally to this work.

Laura Fugazzola

laura.fugazzola@unimi.it

1 Division of Pathology, Fondazione IRCCS Ca' Granda Ospedale Maggiore Policlinico, Milano, Italy

2 Department of Pathophysiology and Transplantation, Università degli Studi di Milano, Milan, Italy

3 Department of Biomedical, Surgical and Dental Sciences, Università degli Studi di Milano, Milan, Italy

4 Division of Endocrine and Metabolic Diseases, IRCCS Istituto Auxologico Italiano, Milano, Italy
Conclusions The PTC-MA assay is a novel MA test, which is able to detect fusion genes generated by genomic rearrangements concomitantly with the analysis of hotspot point mutations, thus allowing the evaluation of key diagnostic, prognostic, and therapeutic markers of PTC in a single experiment without any informatics analysis. As the assay is sensitive, robust, easily achievable, and affordable, it is suitable for the diagnostic practice. Finally, the PTC-MA assay can be easily implemented and updated by adding novel genetic markers, according to clinical requirements.

Keywords Papillary thyroid carcinoma $\cdot$ MassARRAY • $\mathrm{RET} \cdot \mathrm{NTRK} 1 \cdot \mathrm{BRAF} \cdot \mathrm{TERT}$

\section{Introduction}

Papillary thyroid cancer (PTC) accounts for approximately $80 \%$ of thyroid cancers with an increasing prevalence over the last decade [1]. The extensive characterization of PTC reported in The Cancer Genome Atlas allowed the reclassification of PTC cases into molecular subtypes that better reflect their differential properties improving the pathological classification and clinical surveillance [2]. Given the established relevance of molecular characterization, clinical diagnosis is now facing the need for cost and time-effective techniques to investigate multiple molecular markers.

In the present study, we propose the mass spectrometry array (MassARRAY, MA) as a rapid, cost-effective, and sensitive method for the analysis of multiple hotspot point mutations concomitantly with fusion genes typical of PTC, in a single experiment. The procedure requires minimal amounts of material, representing an advantage for routine. 
Indeed, DNA fragments analyzed by MA are short, making it a useful technique for assessment of nucleic acid even from formalin-fixed paraffin-embedded (FFPE) samples. Furthermore, MA is a quantitative method, which provides data on the percentage of mutated allele and allows to identify mutations at low frequencies (5-10\%) [3].

Exploiting the MA genotyping platform we designed an assay, the "PTC-MA assay", to detect simultaneously 13 hotspot point mutations and six recurrent rearrangements, using both genomic DNA and cDNA from tumor samples. Detection of these fusion genes is possible because, although they derive from chromosome rearrangements that can involve different breakpoints at the DNA level, they generate identical fusion products at the transcription level.

\section{Materials and methods}

\section{Samples}

The following cases were evaluated:

a. 23 non-consecutive PTCs harboring common genetic alterations (BRAFV600E, TERT G228A, TERT G250A, NRAS Q61R, NRAS Q61K, HRAS Q61K, RET/PTC1, RET/PTC3) recruited at Fondazione IRCCS Ca' Granda (Table 1). All the alterations of the PTC samples were previously detected by Sanger sequencing, as already reported [4-6]. Samples were frozen tissues in 12 cases, and formalin-FFPE tissues in 11 cases. Five cases were analyzed starting from both frozen and FFPE samples (cases \#1, 2, 5, 6 and $8)$. For frozen samples, tumors $<1.5 \mathrm{~cm}$ were microdissected to ensure high tumor tissue content; in larger tumors, the core of the sample was macroscopically dissected. For FFPE samples, hematoxylin and eosin sections were evaluated to ensure a tumor cell content of at least $70 \%$. To evaluate the accuracy of PTC-MA assay in quantifying mutated alleles, in samples \#1 and \#10, BRAF V600E mutation was assessed also by pyrosequencing (CEIVD Anti-EGFR MoAb response BRAF statusDiatech Pharmacogenetics s.r.l., Jesi, Italy).

b. Three blood and 23 normal thyroid tissues contralateral to the tumor (12 frozen and 11 FFPE) served as negative controls.

c. Positive controls, to validate detection of rare mutations not present in our PTC series: four FFPE lung cancer samples, two harboring mutations AKT1 E17K and PIK3CA E542K (cases \#28 and \#31, Table 1) previously detected by the CE-IVD MassArray Dx lung panel (Diatech Pharmacogenetics, Italy) and two with KRAS G12V and G13C (cases
\#29 and \#30, Table 1) previously assessed by pyrosequencing with the CE-IVD Anti-EGFR MoAb response KRAS status (Diatech Pharmacogenetics, Italy). Unfortunately, no positive controls were available for the EIF1AX mutation.

d. Commercial DNA reference standards HORIZON (Cambridge, UK), harboring the BRAF V600E or the NRAS Q61R mutations at known percentages (10 and $5 \%$ ), to study the sensitivity of the PTC-MA assay in detecting point mutations.

e. Positive controls to validate detection of fusion genes not present in the PTC series: four mouse NIH 3T3 fibroblasts cell lines, transfected with human DNA harboring the fusion genes, TRK, TRK-T1, TRK-T3, and RET/PTC2 [7-10]. These positive controls were also exploited to test the sensitivity of the PTC-MA assay in detecting fusion genes.

The study was approved by the Ethical Committee of the Institution involved.

\section{DNA/RNA extraction, and reverse transcription}

Genomic DNA was extracted from either frozen, FFPE tissues (Puregene ${ }^{\circledR}$ Core Kit A, Qiagen, Germantown, MD, USA), peripheral blood samples (Illustra Nucleon Bacc2, GE Healthcare, Barrington, IL, USA).

Total RNA was extracted from frozen or FFPE tissue samples using a Trizol-based commercial kit (Thermo Fisher, Waltham, MA, USA), and from peripheral leukocytes by the PAX gene Blood RNA System (PreAnalytiX, Hombrechtikon, Switzerland). One microgram of each RNA sample was reverse-transcribed using a Superscript reverse transcriptase II Kit (Thermo Fisher), with a hexamer mixture as primers. $\beta$-actin amplification from the cDNA was performed as an internal quality control. The optimal amount of DNA and cDNA for MA analysis is $30 \mathrm{ng}$; however, when the genetic material is limited, the system can work with $10 \mathrm{ng}$ of starting material, as in the manufacturer's protocol [11].

DNA and cDNA of cell lines were provided by Istituto Nazionale Tumori of Milan.

\section{PTC-MA assay}

Thirteen hotspot mutations and the six recurrent fusion genes, representing the most frequently observed in PTC [2], were included in the PTC-MA assay (Supplemental Table 1).

Three multiplex polymerase chain reaction (PCR) reactions were designed: Mix 1 was performed using DNA and with intronic/exonic PCR primers for point mutations identification, since the primers for TERT, EIFIAX and 


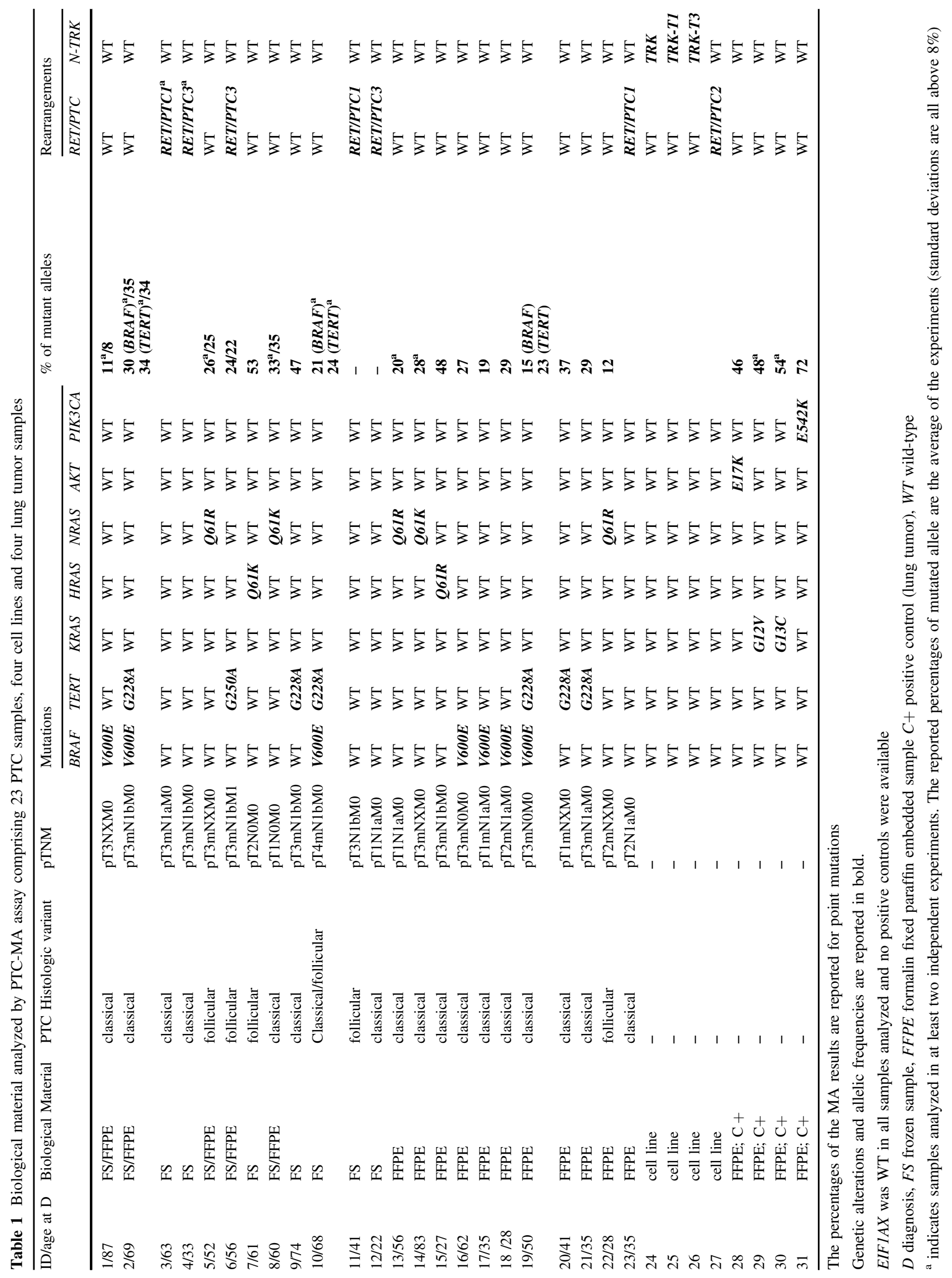




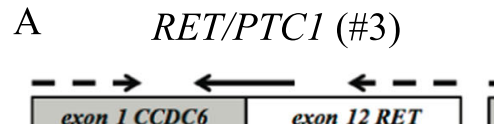

RET/PTC2 (\#27)

RET/PTC3 (\#4)
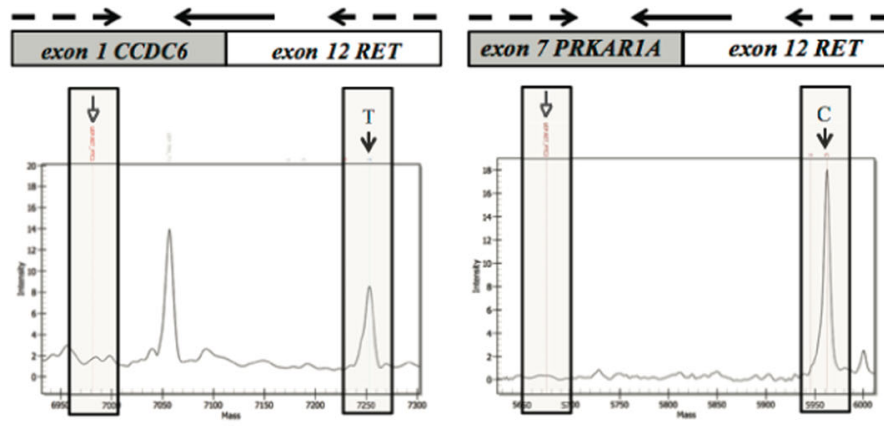

TRK (\#24)

TRK-T1 (\#25)
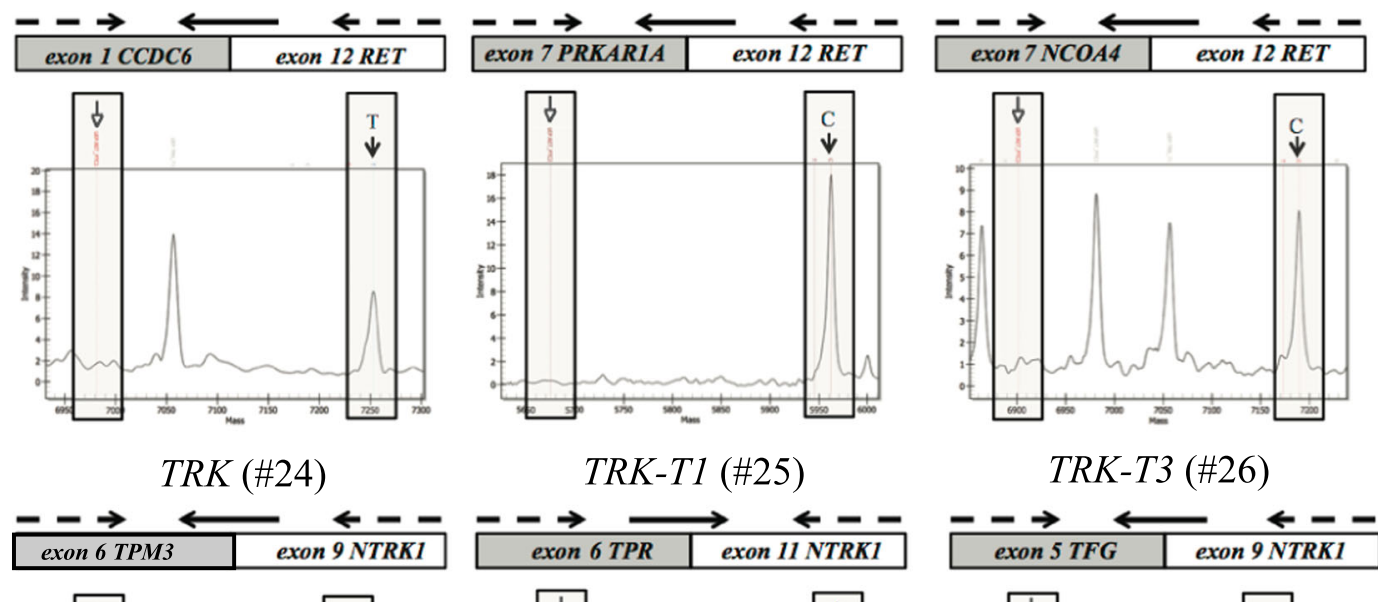

TRK-T3 (\#26)
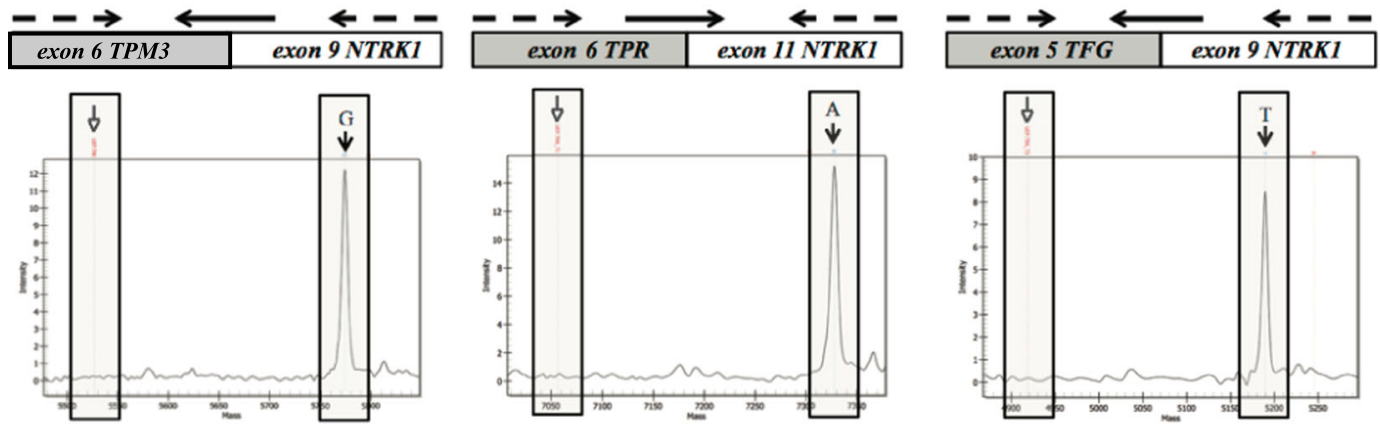

B

BRAF V600E (\#2)
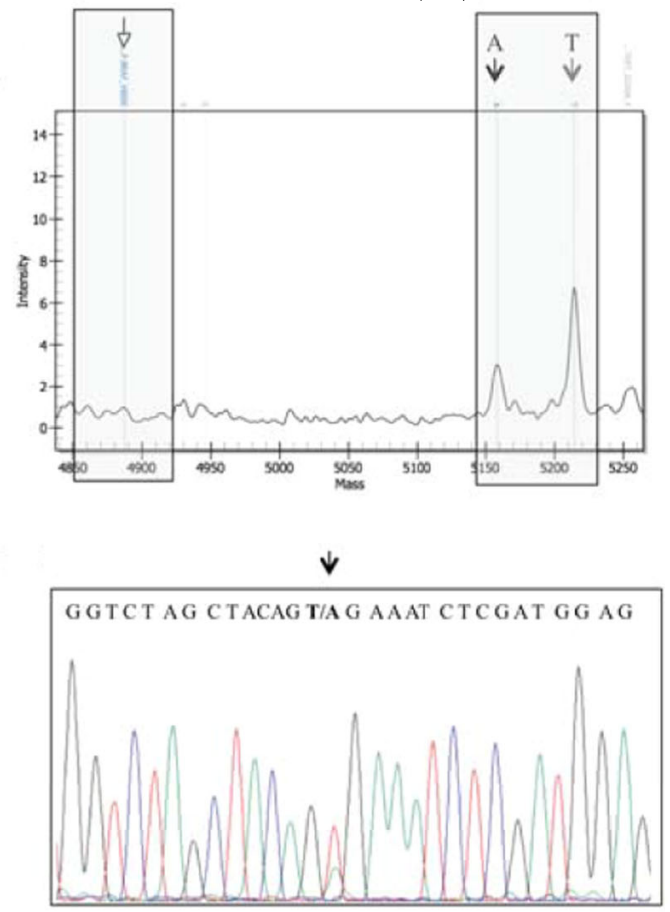
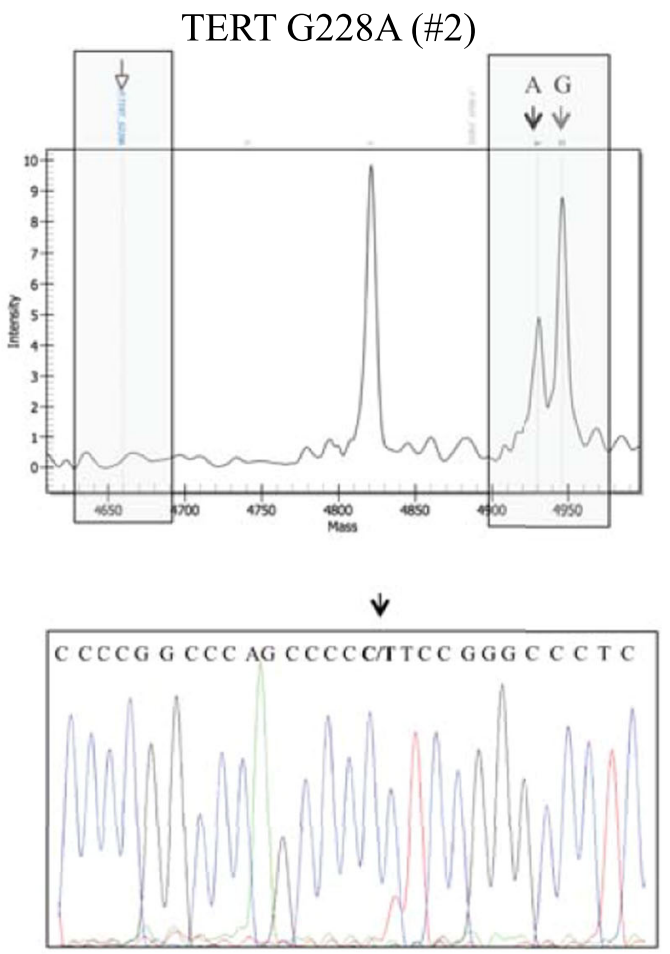

$A K T 1$ were located in non-coding sequences. Mixes 2 and 3 were used to analyze cDNA, with exonic primers for the identification of both point mutations located within exons and fusion genes. For fusion genes, PCR primers were designed in order to obtain a product only in the presence of the rearrangement. Extension primers spanned the fusion 
Fig. 1 MA spectra for gene rearrangements and hotspot mutations. a Schematic representation of PCR and extension primers for the detection of fusion genes and representative MA spectra. The chromosomal regions involved in the formation of fusion genes are indicated in white (RET and NTRK1) and gray (CCDC6, NCOA4, TPM3, $T P R, P R K A R I A$, and TFG), and are reported on the top of the corresponding MA spectrum. The positions of the PCR and extension primers are indicated by dotted and black arrows, respectively. In each spectrum, the empty arrow indicates the position of the unextended primer (when the fusion gene is absent); the black arrow points to the position of the extension product, in presence of the fusion gene. The spectra of RET/PTC1 and RET/PTC3 were generated from the positive tissue samples, (\#3 and \#4, Table 1), and the spectra of RET/PTC2, $T R K, T R K-T 1$ and TRK-T3 were generated from corresponding positive NIH 3T3 cell lines (\#24-27, Table 1). b MA spectra of the coexistent BRAF V600E and TERT G228A mutations and the corresponding Sanger sequences in sample \#2. On the top, the MA spectra for both $B R A F \mathrm{~V} 600 \mathrm{E}$ and TERT G228A. In each spectrum, the black arrow indicates the mutated allele, the gray arrow the wild-type allele, and the empty arrow indicates the position of the extension primer. The corresponding electropherograms obtained by Sanger sequencing are reported below. Black arrows point to the mutated bases. For TERT assays, MA and Sanger sequencing were designed to assess the reverse and forward strands of the gene, respectively

gene transcripts and included parts of both genes. With the aim to minimize the number of reaction mix needed to complete the PTC-MA assay, in order to reduce costs and time of execution, some single nucleotide variant (4 out of 9) were analyzed together with some fusions starting from cDNA. The multiplexing scheme, the PCR conditions and the primers sequences are listed in Supplemental Table 1.

DNA/cDNA were used for PCR, SAP (shrimp alkaline phosphatase), and single-base extension (SBE) reactions; SBE products were analyzed using MassARRAY Typer 4.0 software (all Agena Bioscience, San Diego, CA, USA), following manufacturer's protocol.

\section{Results}

The effectiveness and specificity of the multiplex reactions were firstly determined analyzing all mutations and rearrangements on negative control samples (23 normal thyroid tissue samples, and 3 blood samples). As expected, all controls were wild-type and no PCR/extension products were obtained using the fusion gene-specific primers, indicating the absence of rearrangements (data not shown).

To optimize the multiplex PCR panel, primers were designed to detect 4 out of 9 exonic point mutations on cDNA (Supplemental Table 1). This was possible, since we previously verified that in two PTC samples (cases \#2 and 5), harboring BRAF V600E and NRAS Q61R respectively, the percentage of mutated alleles found in DNA or cDNA were similar (Supplemental Fig. 1), demonstrating that the reaction template (DNA or cDNA) did not influence the point mutations detection.
To test possible differences in the results due to sample type (frozen or FFPE), the PTC-MA assay was performed in five cases (\#1, 2, 5, 6 and 8) in both frozen and FFPE samples, giving superimposable percentages of mutated alleles, thus indicating that PTC-MA assay is highly reliable also on archival material (Table 1).

The PTC-MA assay was then used to test 23 PTC samples harboring diverse genetic alterations and positive controls. As detailed in Table 1, all the point mutations and gene rearrangements were detected by the PTC-MA assay. The main advantage of this assay is the possibility to test recurrent gene rearrangements with always same fusion products, as those typical of PTC. Notably, all RET and NTRK1 rearrangements included in the assay were clearly identified (Fig. 1a).

As shown in Fig. 1b, the co-occurrence of two genetic alterations was also clearly detected by the PTC-MA assay, as for cases \#2, 10 and 19 (harboring both BRAF V600E and TERT G228A mutations) and for case \#6 (RET/PTC3 rearrangement and TERT G250A mutation).

In addition, for BRAF V600E (cases \#1 and 10) and KRAS mutations (cases \#29 and 30), we found similar percentages of the mutated alleles comparing PTC-MA assay and CE-IVD kits based on pyrosequencing, which is considered a gold-standard method for allelic quantification (Supplemental Table 2).

Finally, the sensitivity of the assay in detecting point mutations was verified by commercial DNA reference standards HORIZON for BRAF V600E and NRAS Q61R (harboring known rates, 10 and 5\%, of mutated allele). As expected [3], the PTC-MA assay was able to detect even $5 \%$ of mutated alleles (Supplemental Fig. 2a). Moreover, we assessed the sensitivity for the detection of fusion genes using the cells lines harboring RET and TRK rearrangements (cases \#24-27; Table 1). By serial dilutions with the tumor sample 28, which is negative for RET and TRK rearrangements, we demonstrated that the PTC-MA assay is able to detect the presence of fusion genes with high sensitivity, also when they were present in 5\% only of the total cDNA (Supplemental Fig. 2b).

\section{Discussion}

Reliable, robust and affordable technology for genetic characterization of cancers is important in supporting routine clinical practice. The MA technology has recently been approved for clinical diagnosis, allowing the identification of mutations with high sensitivity [3, 12, 13].

Here, we report a novel MA assay, the PTC-MA assay, able to detect 13 point mutations and six gene rearrangements typical of PTC in a single experiment, thereby saving time (2 working-days) and reducing costs (approximately 
60 euros per sample). It is worth to note that this PTC-MA assay was developed neither to search for novel genetic alterations in PTC, nor to test for all known genetic alterations, but to detect the point mutations and gene fusions most frequently involved in PTC carcinogenesis. Focusing on the clinical utility of the genetic analysis in PTC (more accurate diagnosis, selection of targeted treatments), we believe that NGS technologies or the wide panels of analysis can add little information to our PTC-MA assay, in spite of significantly higher costs comparing with MA [14]. Moreover, NGS is not affordable for small/ medium-sized laboratories, and is thus only rarely applied. Conversely, our PTC-MA assay could be exploited and developed by several laboratories, leading to a better characterization of PTCs, with a positive clinical and therapeutic impact.

PTC-MA assay is appropriate for routine diagnosis because: (i) it is sensitive also for sample with few cancer cells; (ii) it is accurate in determining the percentages of mutated alleles; (iii) it can be easily implemented and updated by adding novel genetic markers, according to clinical requirements.

In conclusion, though these results will be further validated in a larger cohort of cases, the PTC-MA assay is useful and robust for the simultaneous detection of hotspot point mutations and rearrangements in PTC, using DNA and RNA isolated from frozen as well as FFPE samples.

Acknowledgements The authors would like to acknowledge Dr. Angela Greco and Dr. Italia Bongarzone (Istituto Nazionale dei Tumori, Milan, Italy) for the kind contribution of the transfected NIH $3 \mathrm{~T} 3$ cell lines used as positive controls.

\section{Compliance with ethical standards}

Conflict of interest The authors declare that they have no competing interests.

Informed consent Informed consent was obtained from all participants included in the study.

Open Access This article is distributed under the terms of the Creative Commons Attribution 4.0 International License (http://creativecommons.org/licenses/by/4.0/), which permits unrestricted use, distribution, and reproduction in any medium, provided you give appropriate credit to the original author(s) and the source, provide a link to the Creative Commons license, and indicate if changes were made.

\section{References}

1. S. Vaccarella, S. Franceschini, F. Bray, C.P. Wild, M. Plummer, L. Del Maso, Worldwide thyroid-cancer epidemic? The increasing impact of overdiagnosis. N. Engl. J. Med. 375, 614-617 (2016)
2. The Cancer Genome Atlas, Integrated genomic characterization of papillary thyroid carcinoma. Cell 9, 676-690 (2014)

3. M. Ibarrola-Villava, T. Fleitas, M.J. Llorca-Cardeñosa, C. Mongort, E. Alonso, S. Navarro, O. Burgues, A. Vivancos, J.M. Cejalvo, J.A. Perez-Fidalgo, S. Roselló, G. Ribas, A. Cervantes et al. Determination of somatic oncogenic mutations linked to target- based therapies using MassARRAY technology. Oncotarget 7, 22543-22555 (2016)

4. M. Muzza, C. Colombo, S. Rossi, D. Tosi, V. Cirello, M. Perrino, L.S. De, E. Magnani, E. Pignatti, B. Vigo, M. Simoni, G. Bulfamante, L. Vicentini et al. Molecular and cellular endocrinology telomerase in differentiated thyroid cancer: promoter mutations, expression and localization. Mol. Cell Endocrinol. 399, 288-295 (2015). Elsevier Ireland Ltd

5. L. Fugazzola, D. Mannavola, V. Cirello, G. Vannucchi, M. Muzza, L. Vicentini, P. Beck-peccoz, BRAF mutations in an Italian cohort of thyroid cancers. Clin. Endocrinol. (Oxf.) 61, 239-243 (2004)

6. M. Muzza, D.D. Innocenti, C. Colombo, M. Perrino, E. Ravasi, S. Rossi, V. Cirello, P. Beck-Peccoz, M.G. Borrello, L. Fugazzola, The tight relationship between papillary thyroid cancer, autoimmunity and inflammation: clinical and molecular studies. Clin. Endocrinol. (Oxf.) 72, 702-708 (2010)

7. A. Greco, R. Orlandi, C. Mariani, C. Miranda, M.G. Borrello, A. Cattaneo, S. Pagliardini, M.A. Pierotti, Expression of TRK-T1 oncogene induces differentiation of PC12 Cells. Cell Growth Differ. 4, 539-546 (1993)

8. I. Bongarzone, M.G. Monzini, C. Borrello, G. Carcano, G. Ferraresi, E. Arighi, P. Mondellini, G. Della Porta, M.A. Pierotti, Molecular characterization of a thyroid tumor-specific transforming sequence formed by the fusion of ret tyrosine kinase and the regulatory subunit RICL of cyclic AMP-dependent protein kinase A. Mol. Cell Biol. 13, 358-366 (1993)

9. A. Greco, C. Mariani, C. Miranda, A. Lupas, S. Pagliardini, M. Pomati, M.A. Pierotti, The DNA rearrangement that generates the TRK-T3 oncogene involves a novel gene on chromosome 3 whose product has a potential coiled-coil domain. Mol. Cell Biol. 15, 6118-6127 (1995)

10. M. Butti, I. Bongarzone, G. Ferraresi, P. Mondellini, M. Borrello, M. Pierotti, A sequence analysis of the genomic regions involved in the rearrangements between TPM3 and NTRK1 genes producing TRK oncogenes in papillary thyrid carcinomas. Genomics 28, 15-24 (1995)

11. S. Gabriel, L Ziaugra, D. Tabbaa SNP Genotyping Using the Sequenom MassARRAY iPLEX Platform. Curr. Protoc. Hum. Genet 60, 1-16 (2009).

12. T. Fleitas, M. Ibarrola-Villava, G. Ribas, A. Cervantes, MassARRAY determination of somatic oncogenic mutations in solid tumors: Moving forward to personalized medicine. Cancer Treat. Rev. 49, 57-64 (2016)

13. H.-X. Tian, X.-C. Zhang, Z. Wang, J.-G. Chen, S.-L. Chen, W.-B. Guo, Y.-L. Wu, Establishment and application of a multiplex genetic mutation-detection method of lung cancer based on MassARRAY platform. Cancer Biol. Med. 13, 68-76 (2016)

14. S. Sciacchitano, L. Lavra, A. Ulivieri, F. Magi, G.P. De Francesco, C. Bellotti, L.B. Salehi, M. Trovato, C. Drago, A. Bartolazzi, Comparative analysis of diagnostic performance, feasibility and cost of different test-methods for thyroid nodules with indeterminate cytology. Oncotarget 8(30), 49421-49442 (2017) 